\title{
A Method of Angle Error Extraction for BPSK Modulated Signals Based on Instantaneous Autocorrelation
}

\author{
SONG Xiao-rui ${ }^{1}$, ZHENG Hai-xin ${ }^{2}$ and ZENG Chuang-zhan ${ }^{1}$ \\ ${ }^{1}$ Department of Graduate Management, Equipment Academy, 101416, Beijing, China \\ 2 Department of Optical and Electronic Equipment, Equipment Academy, 101416, Beijing, China
}

\begin{abstract}
In this paper, a method of angle error extraction for binary phase-shift keying (BPSK) modulated signals based on instantaneous autocorrelation is proposed for the monopulse angle tracking problem of BPSK signal under a low bit signal to noise ratio (SNR). It reduces the effect of noise on the angle error extraction efficiently by using the characteristics of the instantaneous autocorrelation of noise. The effectiveness and reliability of the theoretical algorithm are proved by MATLAB simulation. As simulation results shown, this method has a good performance under ideal Gaussian noise background. When bit SNR is greater than $2 \mathrm{dBHz}$, the relative error of the estimation results can be less than $5 \%$.
\end{abstract}

\section{Introduction}

The angle error extraction of BPSK signal is a process of computing the ratio between the amplitude of sum signal and different signal by a series of signal processing $[1,2]$. The sum and different signals are received by a Cassegrain antenna for target tracking. The angle error extraction is an important part of the monopulse technology, which is widely used in target tracking in aerospace, communications, modern search radar, radio astronomy, sonar, optical tracking and other fields.

There are two conventional methods of angle error extraction of BPSK signal so far. The first one is based on envelope detection [3, 4], that is, by intercepting a small portion of the wideband signal to make it into a narrowband signal which can be used to estimate the angle error by amplitude detection. This method is a noncoherent demodulation and ineffective under low SNR conditions. The second one is based on I/Q processor [5], that is, by using a PLL to recover the carrier as a local oscillation signal to finish the synchronous detection with which the amplitude modulated information can be recovered to estimate the angle error. This method is a coherent demodulation with a lower threshold than former one and has a widely use in digital monopulse receivers. However, it will be seriously affected by correlated noise in sum and different signals and the precision of angle error estimations will worsen quickly under a low SNR.

Aimed at solving the problems of angle error extraction under low SNR conditions and estimation precision seriously affected by correlated noise in sum and different signals, this paper carry out a method to implement the angle error extraction of BPSK signal under a low SNR based on instantaneous autocorrelation signal processing.

\section{Signal instantaneous autocorrelation characteristics}

The signal instantaneous autocorrelation is defined as a product of in-phase component and quadrature component $[6,7]$ :

$$
S T(t)=s_{I}(t) \cdot s_{Q}(t)
$$

From the properties of Hilbert transform, the phase difference between in-phase component and orthogonal component is $90^{\circ}$, that is, a quarter of a signal period delay, $\tau_{0}=T / 4$, and $T$ is a signal period. Thus, signal instantaneous autocorrelation is equivalent to signal autocorrelation with a fixed delay.

The received signal is given as:

$$
r(t)=s(t)+n(t)
$$

Then we have autocorrelation of received signal as follows,

$$
\begin{aligned}
Y T_{r}(t, \tau) & =r(t) \cdot r^{*}(t-\tau) \\
& =s(t) s^{*}(t-\tau)+s(t) n^{*}(t-\tau) \\
& +n(t) s^{*}(t-\tau)+n(t) n^{*}(t-\tau)
\end{aligned}
$$

where noise $n(t)$ is incoherent with the signal. When $\tau \neq 0$, we have

$$
E\left[n(t) \cdot n^{*}(t-\tau)\right]=0
$$

then (3) can be expressed as:

$$
Y T_{r}(t, \tau)=s(t) s^{*}(t-\tau)
$$


From the above derivation, it is can be known that the autocorrelation of received signal can reduce noise effect efficiently. And signal instantaneous autocorrelation can be given as:

$$
\begin{aligned}
S T(t) & =s_{I}(t) \cdot s_{Q}(t) \\
& =s(t) \cdot s^{*}\left(t-\tau_{0}\right) \\
& =Y T_{r}\left(t, \tau_{0}\right)
\end{aligned}
$$

Equation(6) shows that the substance of signal instantaneous autocorrelation is an autocorrelation with a fixed delay, which is a quarter of a signal period. Therefore, the noise effect can be reduced efficiently after the instantaneous autocorrelation signal processing to provide a better SNR condition to angle error estimation.

\section{Angle error extraction based instantaneous autocorrelation}

Section II shows that signals can have a better SNR condition for follow processing after the instantaneous autocorrelation. With this feature, this paper provides a method of angle error extraction based on instantaneous autocorrelation. The process of angle error extraction is shown in Fig. 1.

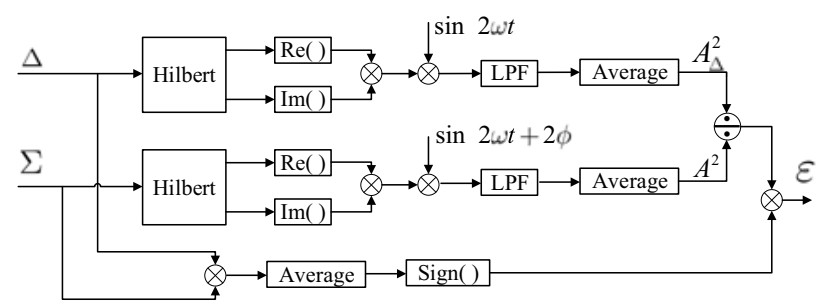

Figure 1. Block diagrams of the process of angle error extraction based on instantaneous autocorrelation.

The sum and different signals with ideal Gaussian noise are given as:

$$
\begin{aligned}
\Sigma & =A m(t) \cos (\omega t)+n_{s} \\
\Delta_{a z} & =A_{a z} m(t) \cos \left(\omega t+\phi_{A}\right)+n_{a z} \\
\Delta_{a z} & =A_{a z} m(t) \cos \left(\omega t+\phi_{A}\right)+n_{a z}
\end{aligned}
$$

where, $m(t)$ is the encoded data with values of +1 or -1 ; $\Sigma$ is the sum signal; $\Delta_{a z}$ is the azimuth difference signal; $\Delta_{e l}$ is the elevation difference signal. And $\phi_{A}$ is the phase difference between the azimuth different and the sum signals; $\phi_{E}$ is the phase difference between the elevation different and the sum signals. In addition, $A$ stands for the amplitude of sum signal, $A_{a z}$ for the amplitude of the azimuth different signal, and $A_{e l}$ for the amplitude of the elevation different signal. $n_{s}, n_{a z}$ and $n_{e l}$ are the noise in the sum, azimuth different and elevation different signal, separately. Therefore, according to the basic principle of monopulse angle tracking system, we can get the azimuth and the elevation angle error:

$$
\begin{aligned}
\varepsilon_{a z} & =A_{a z} / A \\
\varepsilon_{e l} & =A_{e l} / A
\end{aligned}
$$

Computing the Hilbert transform of the sum and different signals, we can take the real parts as in-phase components and the imaginary parts as quadrature components:

$$
\begin{gathered}
\Sigma_{S I}=A m(t) \cos (\omega t)+n_{s} \\
\Delta_{a z_{-} S I}=A_{a z} m(t) \cos \left(\omega t+\phi_{A}\right)+n_{a z} \\
\Delta_{e l_{-} S I}=A_{e l} m(t) \cos \left(\omega t+\phi_{E}\right)+n_{e l} \\
\Sigma_{S Q}=A m(t) \sin (\omega t)+n_{s}{ }^{*} \\
\Delta_{a z_{-} S Q}=A_{a z} m(t) \sin \left(\omega t+\phi_{A}\right)+n_{a z}{ }^{*} \\
\Delta_{e l_{-} S Q}=A_{e l} m(t) \sin \left(\omega t+\phi_{E}\right)+n_{e l}{ }^{*}
\end{gathered}
$$

where, $\Sigma_{S I}, \Delta_{a z S I}$ and $\Delta_{e l S I}$ are the in-phase components of the sum, azimuth different and elevation different signals, separately. Similarly, $\Sigma_{S Q}$, $\Delta_{a z} S Q$ and $\Delta_{e l s Q}$ are the quadrature components of the sum, azimuth different and elevation different signal separately.

The signals after instantaneous autocorrelation are:

$$
\begin{aligned}
S T_{\Sigma}= & A^{2} \sin (2 \omega t) \\
& +n_{s}{ }^{*} A m(t) \cos (\omega t) \\
& +n_{s} A m(t) \sin (\omega t)+n_{s}{ }^{*} n_{s} \\
S T_{\Delta_{a z}}= & A_{a z}{ }^{2} \sin \left(2 \omega t+2 \phi_{A}\right) \\
& +n_{a z}{ }^{*} A_{a z} m(t) \cos \left(\omega t+\phi_{A}\right) \\
& +n_{a z} A_{a z} m(t) \sin \left(\omega t+\phi_{A}\right)+n_{a z}{ }^{*} n_{a z} \\
S T_{\Delta_{e l}}= & A_{e l}{ }^{2} \sin \left(2 \omega t+2 \phi_{E}\right) \\
& +n_{e l}{ }^{*} A_{e l} m(t) \cos \left(\omega t+\phi_{E}\right) \\
& +n_{e l} A_{e l} m(t) \sin \left(\omega t+\phi_{E}\right)+n_{e l}{ }^{*} n_{e l}
\end{aligned}
$$

Since the results of noise autocorrelation are zero with non-zero delay, we can get

$$
E\left(n^{*} n\right)=0
$$

The square value of sum and different amplitude can be solved by down converting process of (18), (19) and (20). The solution functions are:

$$
\begin{aligned}
\Sigma_{A M P} & =E\left[S T_{\Sigma} \cdot \sin (2 \omega t)\right] \\
& =A^{2} \\
\Delta_{a z_{-} A M P} & =E\left[S T_{\Delta_{a z}} \cdot \sin \left(2 \omega t+\phi_{A}\right)\right] \\
& =A_{a z}{ }^{2} \\
\Delta_{\mathrm{el} \_A M P} & =E\left[S T_{\Delta_{e l}} \cdot \sin \left(2 \omega t+\phi_{E}\right)\right] \\
& =A_{e l}{ }^{2}
\end{aligned}
$$

To be here, the local oscillator signals used in down conversion are from numerically controlled oscillators(NCO) in sum, azimuth different and elevation different channels separately. The difference between the three lies in the initial phases.

The sign of the angle error can be differentiated by the functions as follows:

$$
\operatorname{Symbol}_{a z}=\operatorname{sign}\left(\Delta_{a z} \cdot \Sigma\right)
$$




$$
\operatorname{Symbol}_{e l}=\operatorname{sign}\left(\Delta_{e l} \cdot \Sigma\right)
$$

At last, the azimuth and elevation angle error are estimated as:

$$
\begin{aligned}
& \varepsilon_{a z}=\operatorname{Symbol}_{a z} \cdot \sqrt{\Delta_{a z_{-} A M P} / \Sigma_{A M P}} \\
& \varepsilon_{e l}=\operatorname{Symbol}_{e l} \cdot \sqrt{\Delta_{\mathrm{el} \_A M P} / \Sigma_{A M P}}
\end{aligned}
$$

\section{Simulation and Analysis}

In this section, a simulation will be carried out with Matlab to proof the validity of the algorithm. Because the methods of azimuth and elevation angle error extraction are the same [8-10], only the azimuth angle error extraction will be simulated here. And the elevation angle error is estimated in the same way. The simulation parameter settings are as follows:

The code rate of BPSK signal: $R_{b}=2 \mathrm{Mbps}$

The carrier frequency: $f_{c}=70 \mathrm{MHz}$

The sampling frequency: $f_{s}=60 \mathrm{MHz}$

The truth value of azimuth angle error: ${ }_{a z}=-0.5$

The baseband signal is a sequence of 0 and 1 generated at random, and the noise in signals is ideal Gaussian noise.

100 times Monte Carlo experiments are conducted to estimate the azimuth angle error under different SNR conditions. In the experiments, the bit SNR varies from $6 \mathrm{dBHz}$ to $16 \mathrm{dBHz}$ in a $0.5 \mathrm{dBHz}$ step. The result of the simulation is shown in Fig. 2.

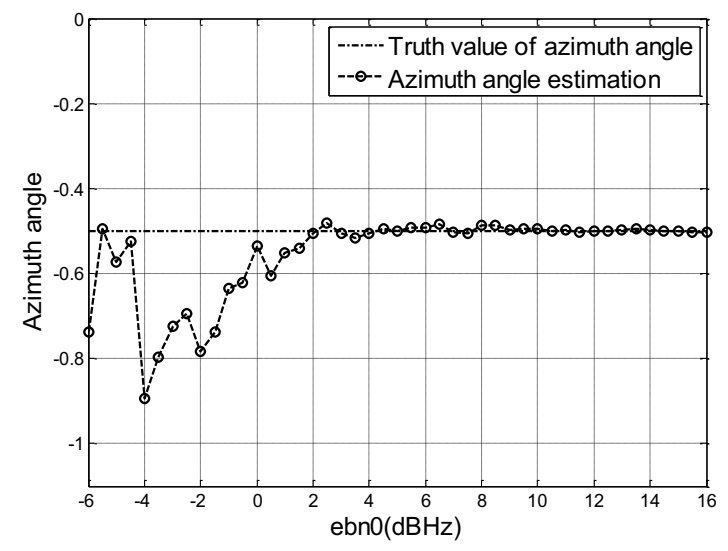

Figure 2. Azimuth angle error estimations under different bit SNR conditions.

It is shown in Fig. 2 that, all the relative errors between the azimuth angle error estimations and the truth values are less than $5 \%$ when $E b / N_{0}>2 \mathrm{dBHz}$. The estimation precision can meet the needs of monopulse tracking system [11, 12]. Under the same simulation conditions, the comparison between different methods of angle error extraction is shown in Fig. 3 as follows.

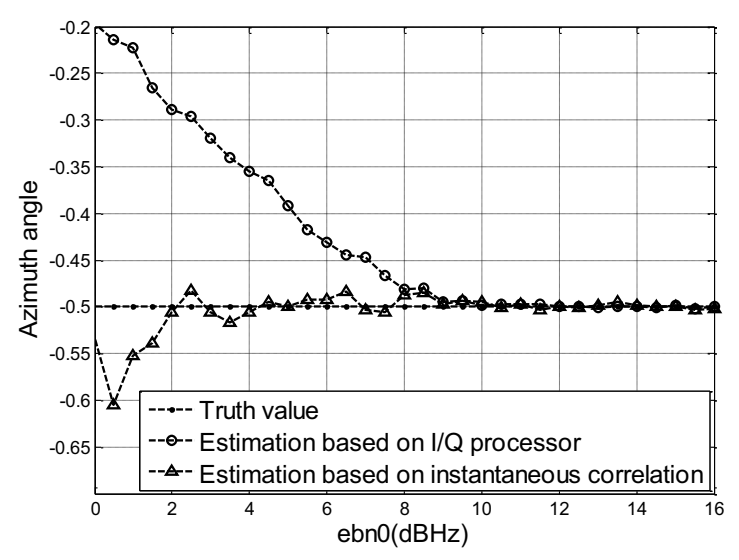

Figure 3. Angle error estimations of two methods.

Fig. 3 shows that, the threshold of the angle error extraction based on $\mathrm{I} / \mathrm{Q}$ processor is about $8 \mathrm{dBHz}$, but it of the angle error extraction based on instantaneous autocorrelation is about $2 \mathrm{dBHz}$. In other words, the threshold has a $6 \mathrm{dBHz}$ decrease so that the performance of angle error extraction can improve significantly. In addition, unlike extraction method based on I/Q processor, the precision of angle error extraction based on instantaneous autocorrelation is not affected by noise independent or not.

The derivation in Section III shows that there are two error sources in the angle error extraction based on instantaneous autocorrelation. The first one is symbol estimation error, and the second one is the absolute value estimation error. The analysis of the two error sources is as follows.

Firstly, an analysis in symbol estimation error is carried out. Under different SNR conditions, the number of false symbols in 100 times Monte Carlo experiments is counted and the result is shown in Fig. 4.

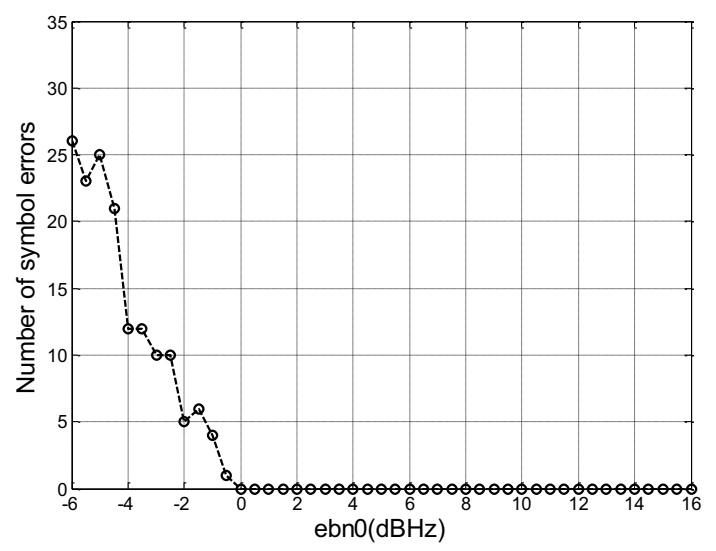

Figure 4. The statistic of false symbol estimations.

In Fig. 4, the number of false symbols is zero when $E b / N_{0}>0 \mathrm{dBHz}$, which shows that there is no symbol estimation error under this condition. When $E b / N_{0}<0 \mathrm{dBHz}$, the precision of symbol estimation will begin worsening and the number of false symbols will increase rapidly with the SNR decreasing.

Secondly, an analysis in absolute value estimation error is carried out. Fig. 5 shows the azimuth angle 
absolute value estimations in 100 times Monte Carlo experiments under different SNR conditions.

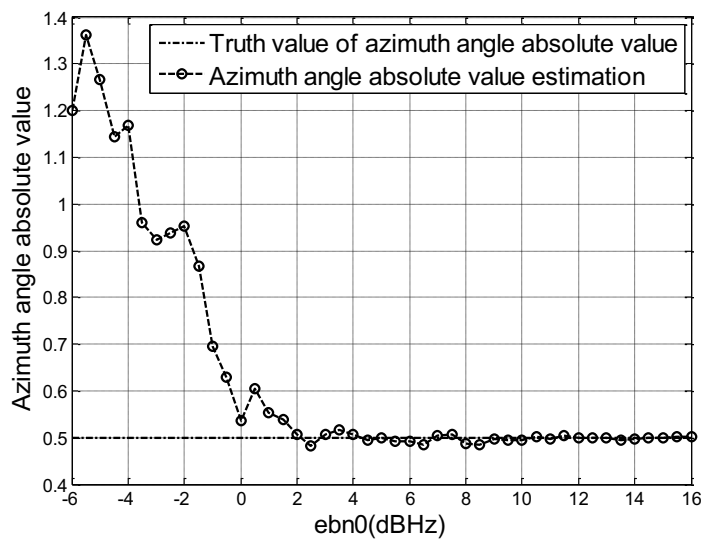

Figure 5. Azimuth angle absolute value estimations.

Fig. 5 shows that the relative errors between the azimuth angle absolute value estimations and the truth values are less than $5 \%$ when $E b / N_{0}>2 \mathrm{dBHz}$, meeting the requirements of angle tracking precision. And the precision cannot be meet with a bit SNR greater than $0 \mathrm{dBHz}$ less than $2 \mathrm{dBHz}$.

To sum up, the threshold of the symbol estimation is about $0 \mathrm{dBHz}$, but the threshold of the absolute value estimation is about $2 \mathrm{dBHz}$. Therefore, the main restriction to the angle error extraction which is based on instantaneous autocorrelation, is the angle error absolute value estimation precision under a low SNR condition.

\section{Conclusions}

In this paper, a method of angle error extraction for BPSK modulated signals is provided. The main innovation point of the method is using the characteristics of the instantaneous autocorrelation of BPSK signals to reduce the effect of noise on the angle error extraction. As a result, the problems of angle error extraction under low SNR conditions and estimation precision seriously affected by correlated noise in the sum and different signals can be solved. However, this method is only apply to the three-channel monopulse angle tracking system, and how to realize the application in the dualchannel or the single channel monopulse angle tracking system remains to be solved.

\section{Acknowledgment}

The authors wish to thank Zhu Jiang, Yan Di, and Li Zibo. This work was supported in part by a grant from the Laboratory of TT\&C, Department of Optical and Electronic Equipment, Equipment Academy.

\section{References}

1. Levanon, Nadav. Radar principles. New York: Wiley-Interscience, 1988, 320 p.1 (1988).
2. Nickel, Ulrich. "Overview of generalized monopulse estimation", Aerospace and Electronic Systems Magazine, IEEE, 21.6 (2006): 27-56.Luigi T.De Luca, Propulsion physics (EDP Sciences, Les Ulis, 2009)

3. Burgos-Garcia, M., et al. "Digital Monopulse Receivers For Phase Modulated Signals." in Radar Conference, 2003. Proceedings of the International, 2003:640-645.

4. Hofstetter, E. M., and D. Delong. "Detection and parameter estimation in an amplitude-comparison monopulse radar.", IEEE Transactions on Information Theory, 15.1(1969):22 - 30.

5. M. Sherman S, K. Barton D. Monopulse Principles and Techniques. Artech House, 2011.

6. ZHOU Min, FENG Quanyuan. "A new feature parameter for MFSK/MPSK recognition." in The 2011 International Conference on Intelligent Science and Information Engineerin (ISIE). Wuhan, IEEE, 2011:21-23.

7. FU Shiping, YANG Li, SHAO Wei. "Digital Signal Modulation Classification Algorithm Based on Instantaneous Parameter". Modern Defence Technology, 2013,41(6):71-75.

8. Conroy, J. T., Joseph L. LoCicero, and Donald R. Ucci. "Communication techniques using monopulse waveforms." In Military Communications Conference Proceedings, 1999. MILCOM 1999. IEEE. Vol. 2. IEEE, 1999.

9. Seifer, Arnold D. "Monopulse-radar angle tracking in noise or noise jamming.", IEEE Transactions on Aerospace \& Electronic Systems, 28.3(1992):622638.

10. Mosca, E. "Angle Estimation in Amplitude Comparison Monopulse Systems.", IEEE Transactions on Aerospace \& Electronic Systems, aes-5.2(1969):205-212.

11. Armstrong, B. C., and H. D. Griffiths. "Use Of Difference Channel Information For Detection In Monopulse Radars.", Radar \& Signal Processing IEE Proceedings-F, 38.3(1991):199 - 210.

12. Nickel, U. "Performance of corrected adaptive monopulse estimation.", Radar, Sonar and Navigation, IEE Proceedings -F, 146.1(1999):17-24. 